\title{
An Economic Approach to Energy Budgets: How Many Resources Should Living Organisms Spare?
}

\author{
Arturo Tozzi \\ Center for Nonlinear Science, Department of Physics, University of North Texas, Denton, Texas, USA \\ 1155 Union Circle, \#311427Denton, TX 76203-5017 USA \\ tozziarturo@libero.it
}

\begin{abstract}
Ramsey's economic theory of saving (RTS) estimates how much of its commodities a nation should save to safeguard the well-being of future generations. Since RTS retains many attractive qualities such as simplicity, strength, breadth and generality, here we ask if it would be useful to investigate biophysical issues. Specifically, we focus on a biological topic that lends itself as a backdrop for the study of the imbalance between intake and expenditure, i.e., the evaluation of the multicellular living organisms' energetic requirements and constraints. Our problem is to find at each time the optimum distribution and the right balance of the cellular energy budget between consumption and storage: how much must a living organism spare to increase its chances of survival over long periods? Suggesting how to find the optimum allocation of the available energy between expenditure and saving at each time, RTS approaches to biological energy budgets may have a wide range of experimental applications, such as: a) optimization of the long-term survival chances of either immortalized cell cultures, or beneficial bacterial colonies and exogenous probiotic mixtures; b) eradication of detrimental biofilms, such as, e.g., heart valves' Streptococcus colonies; c) novel anti-stress and anti-ageing strategies.
\end{abstract}

Keywords: economics; ATP; energy budget; cellular activity; ageing; prebiotic.

\section{INTRODUCTION}

Every living organism requires energy for maintenance, growth and reproduction, since the whole dynamics of cell fate decisions and regulatory networks rely on ATP levels and cellular energy budgets (Kerr et al., 2019). The energy budget provides useful information concerning the current metabolic state of the cell, embryogenesis (Song et al., 2019), dysfunction or dysregulation of relevant signaling pathways (Papkovsky and Zhdanov AV. 2021), aberrant expression/activity of proteins involved in ATP production (Zhdanov et al., 2011), etc. To provide an example, organisms exposed to suboptimal conditions (e.g., starvation, pharmacological treatment, diabetes, cancer) face an a priori cost of fighting stress in terms of metabolic resources (see, e.g., Smolders et al., 2003; Schmidt et al., 2021). The experiments concerning influence of energy availability on regulatory network dynamics usually evaluate how energy budgets affect both the microscopic cell behaviour (Kerr et al., 2019) and the mechanistic connections of sub-organismal processes. However, energy budgets can be calculated not just at the micro-levels of cellular activity, but also at different macrolevels of living systems' activities. For example, the local increases in spike frequency during perceptual and motor activity of the brain requires additional enthalpy consumption and free-energy production: while ATP consumption at the mean rate at $4 \mathrm{~Hz}$ is $3.29 \times 10^{9}$ molecules of ATP/neuron/sec, increased spike frequency has a metabolic cost of 6.5 $\mu \mathrm{mol} / \mathrm{ATP} / \mathrm{gr} / \mathrm{min}$ for each additional spike (Attwell et al., 2001). Furthermore, the metabolic cost of animal growth, which quantifies the amount of energy required to synthesize a unit of biomass, is a key factor for the making of ontogenetic energy budgets (Ferral et al., 2020). Also, energy budgets provide the proper operational framework to examine the acquisition of energy resources at higher degrees of organization, such as those at the population and ecosystem levels (Murphy et al., 2018).

We ask whether the storing capacity of the living organisms' energy pathways can be quantitatively assessed through mathematical models. Here the Ramsey's economic theory of saving (RTS) comes into play (Ramsey 1928), providing a simple and profitable mathematical apparatus generalizable enough to be used for the assessment of energy budgets. "How much of its income should a nation save for the next generations?" The question was put forward and successfully tackled by the versatile 25 years old "economist" Frank Plumpton Ramsey in 1928, two years before his unexpected death (Misak 2020). Inspired by Ramsey's rather general mathematical/economical approach to saving, we will provide an effort to mathematically cope with biological issues related with energy budget. In particular, we will ask to ourselves the following tantamount question: "how much of its energy budget should a living organism save over time for the most efficient long-term survival?". Also, we will tackle the next obvious question: "Why should we use RTS to assess the energy budget of living organisms?" 


\section{RAMSEY'S MODEL: THE ORIGINAL ACCOUNT}

"How much of its output should a nation save over time and across generations?". In touch with the ClassicalUtilitarianism calculus, Ramey believed that an accurate balancing is required between use and storage. He identified the best match between attainable and desirable utility streams over time and across generations (Misak 2020). Indeed, immediate energetic consumption produces utility now but reduces the opportunities in the future, while energetic saving reduces utility now and increases it in the future. Ramsey requires to invest an amount which may have no immediate payoff but may nevertheless produce a big payoff for future generations.

The key elements of RTS are individuals' lifetime well-beings who are subject to resource constraints (Ramsey 1928). The demographic profile over time is taken to be given, such that the future numbers of people are seen as exogenous and predictable (Dasgupta 1969). Since these individuals are considered identical, a single individual at each date may be assumed, removing any distinction between time and generations. Economic uncertainty is not an option, since the economy, assumed to be closed to international trade, is endowed with a single, non-depreciating commodity that can be worked by labour to produce output at each date. Ramsey's approach neglects the differences between distinct kinds of goods and labour, so that we are allowed to simply speak of general quantities such as capital, consumption and labour without considering their particular forms.

Ramsey's model makes other simplifying assumptions (Parfit 1984):

1) The future is assumed to be deterministic and infinitely long.

2) The community goes on forever.

3) The community will be always maintaining the same size. Therefore, the population is assumed to be constant.

4) The community will be always maintaining the same capacity for aversion to labour and enjoyment.

5) The community will be always governed by the same motives in accumulation.

6) Inventions, technical innovations and improvements in organization are not allowed.

7) No misfortunes, such as earthquakes, wars, catastrophes, will occur to sweep away the nation's accumulations.

Since some of the output can be either invested to increase the commodity's stock or consumed immediately, the practical problem is to find the optimum allocation of output at each date between consumption and investment. Ramsey argues that savings plus consumption must equal the income (Ramsey 1928):

$$
\frac{d c}{d t}+x=f(a, c)
$$

where $\mathrm{x}(\mathrm{t})$ is the total rate of consumption or expenditure in a community and $\mathrm{a}(\mathrm{t})$ is the total rate of labour in a community. Further, $\mathrm{c}(\mathrm{t})$ is the capital (i.e., the stock of the commodity that serves to produce output) at time $\mathrm{t}$, while $\mathrm{c}_{0}$ is the given capital with which the nation starts at $t=0, d c / d t$ are the savings and $f(a, c)$ is the income. All the terms $x, a$ and $c$ are functions of one independent variable, the time, with $t \rightarrow \infty$.

A series of mathematical steps leads Ramsey to his main results, i.e., a general rule stating that the rate of saving multiplied by the marginal utility of money should always be equal to the maximum possible rate minus the actual rate of utility enjoyed. The corresponding equation is:

$$
\frac{d c}{d t}=f(a, c)-x=\frac{B-(U(x)-V(a))}{u(x)}
$$

$\mathrm{U}(\mathrm{x})$ is the total rate of utility of a rate of consumption $\mathrm{x}$, while $\mathrm{u}(\mathrm{x})$, which never increases, is the marginal rate of utility of a rate of consumption $x$. We'd like to remind that the economic utility is the satisfaction or benefit derived by consuming a product (Kahneman and Tversky1979). Therefore, the marginal utility of a good/service describes how much pleasure or satisfaction is gained from an increase in consumption (Castro and Araujo 2019). Furthermore, V(a) is the total rate of disutility of a rate of labour a, while $v(a)$, which never decreases, is the marginal rate of disutility of a rate of consumption x. Indeed, labour supply is an exogenously given constant which is unpleasant and characterized by disutility. The difference $\mathrm{U}(\mathrm{x})-\mathrm{V}(\mathrm{a})$ is the net enjoyment per unit of time: this value is a maximum subject to the condition that $\mathrm{x}$ equals what can be produced with a and c. The term $\mathrm{B}$ (Bliss) is the maximum obtainable rate of enjoinment which, with time passing, approaches asymptomatically to a certain finite limit.

After a few steps (see Ramsey 1928), the main equation can be rewritten as follows:

$$
B-W(y)=\frac{d W}{d y}(r c-y)
$$


Where rc is the unearned income, $\mathrm{W}(\mathrm{y})=\mathrm{U}(\mathrm{x})-\mathrm{V}(\mathrm{a})$ is the total marginal utility of an unearned income available for consumption, while $\mathrm{w}(\mathrm{y})$ is the corresponding marginal utility. The term $\mathrm{y}$ stands for $\mathrm{x}-\mathrm{pa}$, i.e., the consumption minus the earned income.

The results achieved by Ramsey display surprising generality. They lead to valuable operational results: given an amount of unearned income available for consumption, the rule allows to estimate the consumed and the saved unearned incomes. As an example, the Ramsey's plot describing the behaviour of long-term communities is illustrated in Figure 1.

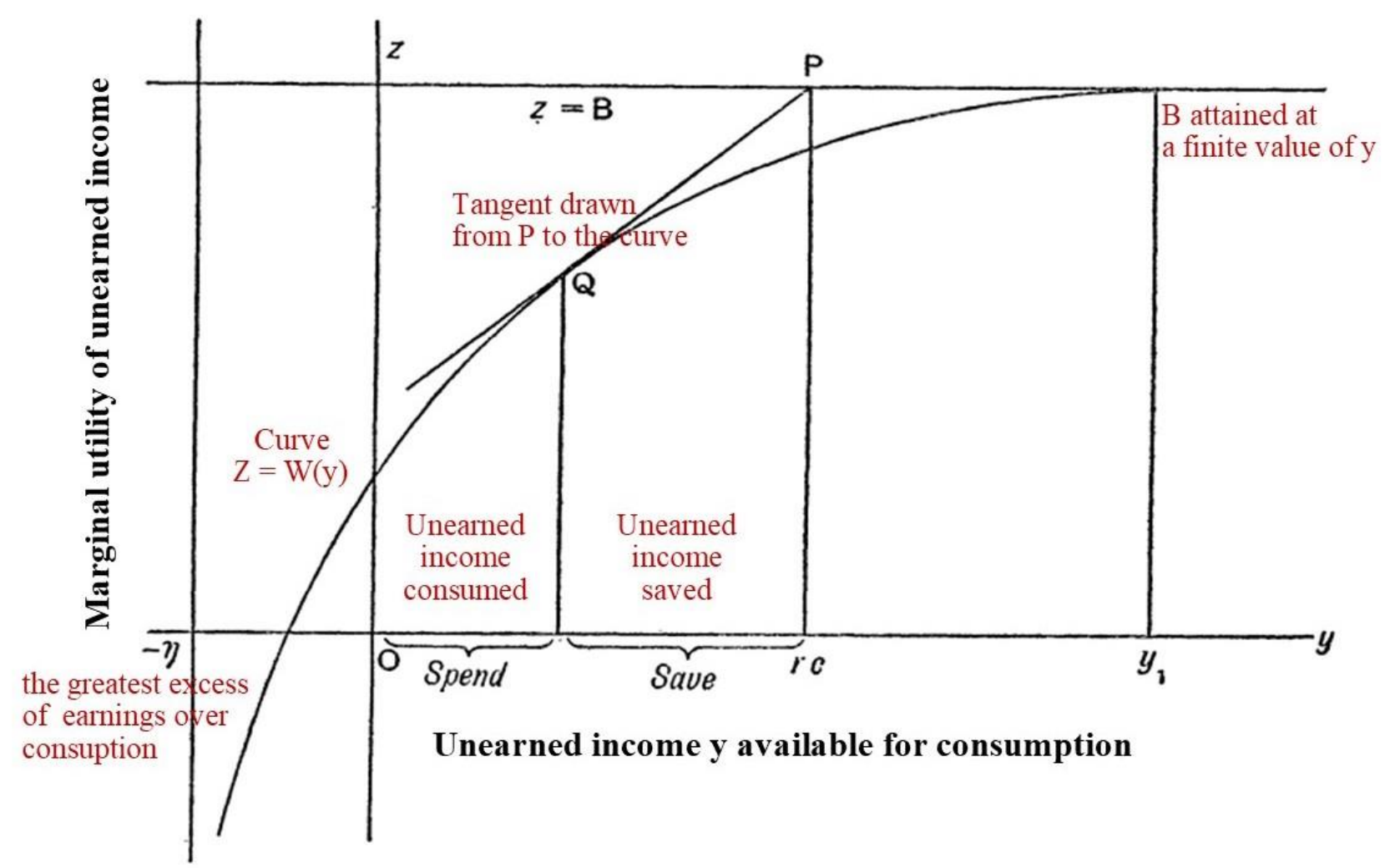

Figure 1. A community which lives forever according to Ramsey's account. The utility is assumed to be an increasing and strictly concave function which is bounded above. Bliss turnpike is beyond our reach, but we can measure how close we are to it to determine the trade-off between consumption and saving at each point of time. Once identified the amount of unearned income available for consumption ( $\mathrm{rc}$ in the Figure), the consumed and the saved unearned incomes can be easily calculated. Modified from Ramsey (1928). 


\section{RAMSEY'S MODEL: THE BIOLOGICAL COUNTERPART}

RTS provides a basic and broad mathematical apparatus for the evaluation of other scientific issues, such as, in our case, living organisms' energy budgets. Our biological problem is to find at each time the optimum distribution between the consumed and the saved energy, or in other words, to determine the right balance between consumption and investment. Our goal is to answer to the question: how much can be spared at a given value of energy?

Starting from the energy available for consumption, the quantity of energy to spare in order to achieve the highest benefit for the future and for the next generations is easily calculated. Erasing any distinction between time passing and generations, living beings are taken to be identical, so that the occurrence of a single individual at each date may be assumed. The intentions are assumed always realized, while the amount of available sources to be always fully deployed and the labour to be fully employed. Since Ramsey does not incorporate uncertainty about future possibilities, we consider individual's life as characterized:

a) By one and only energetic source.

b) By an exogenous constant, termed cellular labour, which produces at each date an output consisting of energy (Gale, 1967, and Brock, 1973).

In touch with RTS, the future survival of living organisms is seen as exogenous and predictable, having the external factors a negligible effect on their survival. The cellular economy is assumed to be closed to energetic changes with other living beings. This means that some of the produced energy can be used to increase the energy stock, while the remainder can be consumed immediately. No mention is made of possible phenotypic, epigenetic, genotypic, evolutionary improvements in cellular capability of labour or ability to achieve a larger quantity of available sources. In touch with Ramsey's account, the anticipated energy production at each moment equals the sum of intended storage and intended consumption. Since the living being is in equilibrium at each moment, the intended saving equals the intended investment at each moment.

The biological counterpart to RTS runs as follows: in a living system (say, a monocellular living organism) the energetic savings plus the consumption must equal the income. Concerning the terminology described in the previous chapter, in our biologic case the capital c stands for the amount of the available energetic source that serves to produce energy, while $\mathrm{x}(\mathrm{t})$ is the total rate of consumption or expenditure inside the cell. Also, $\mathrm{a}(\mathrm{t})$ is the total rate of labour produced by cellular substructures, $\mathrm{c}(\mathrm{t})$ is the capital, i.e., the stock of ATP that serves to produce the required outputs at time $\mathrm{t}$. Then, dc/dt are the savings, while $f(a, c)$ is the income. The most important factor, i.e., $W(y)=U(x)-V(a)$ stands for the living organism's benefits derived from consuming energy. The term B (Bliss) is the maximum obtainable benefit from consuming energy and tends to approach asymptomatically to a certain finite limit. Figure 2 illustrates the biological counterpart of the concave economic function described in the plot of Figure 1.

So, Ramsey's problem can be cast equally as, "how much of a living organism's energetic production should it consume?" The biological consequences of a RTS approach to living organism's energetic dynamics are straightforward. Since the amount of available sources is assumed not to waste or decrease in benefit, the gross energetic investment equals the net energetic investment. If the consumption is lower than the energetic production at a given time, the investment is positive and the amount of the available sources increases; in turn, if the consumption exceeds the energetic production at a given time, the investment is negative and the amount of available sources is doomed to decline. When sources are available, the produced energy is always $>0$. When the use of available energy increases, the resulting benefits are always positive. It is noteworthy that the resulting benefits depend solely on the quantity of available energy. After the consumption of a certain quantity of energy, the resulting benefits reach a plateau, and further increases in energy are not able to improve anymore the benefits. When the benefits are the highest possible, the spared energy is zero, since the tangent parallels the maximum.

The most important consequence of the application of RTS to the energy budget of living organisms is the following: when the available energetic value is known, the consumed and the spared energy can be easily calculated looking at the plot of the concave function (Figure 2). 


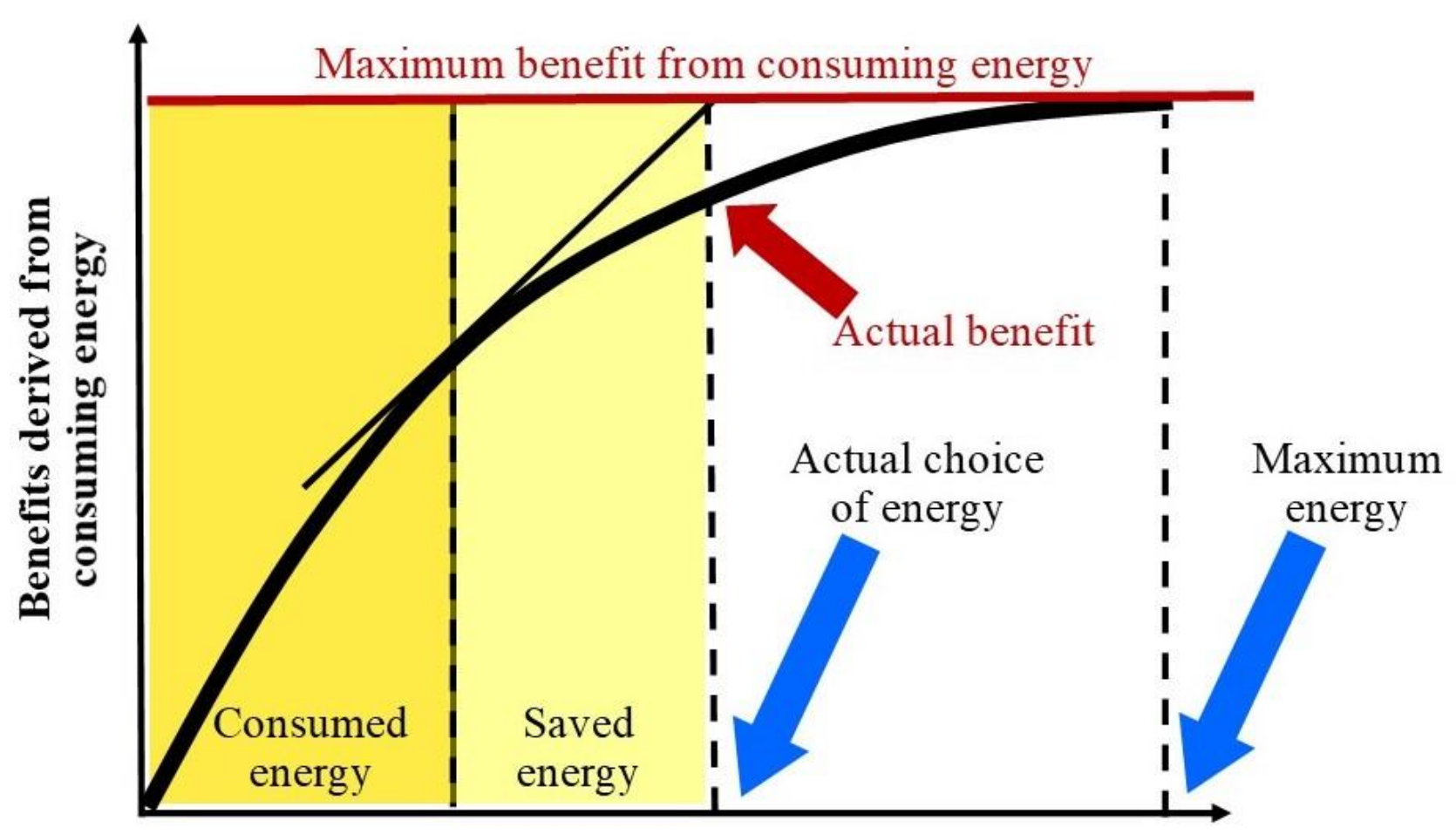

Energy available for consumption

Figure 2. Living organisms' energy budget according to Ramsey's model. In our biological context, the utility stands for the cellular benefits derived by energetic consumption. The curve describes how much benefit is gained by the living organism from an increase in energy consumption. The plot also says that the more is spared, the sooner the highest benefits will be reached, although the actual benefits are scarce. See text for further details.

\section{CONCLUSIONS}

Ramsey's theory of savings (RTS) evaluates how much natural capital a nation should save. Put in terms of maximal utility over the long run, it provides a general scheme which could be profitably used to assess recent hot topics such as exhaustible resources, climate change, nuclear waste disposal (Misak 2020). For our part, our aim was to cast Ramsey's economic problem in terms of: "how much of a living organism's energetic production should it consume?". We found that Ramsey's model can be used to mathematically describe the energy budget of living organisms. The evaluation of energy budgets requires quantities that can be (relatively easily) operationally assessed. For example, the cellular energy budget can be measured through different available techniques that facilitate the comparison of different cells, such as direct measurement, extracellular flux analysis, high-resolution respirometry, fluorescent dye kits (Schmidt et al., 2021), specific bioenergetic signatures such as respiration, glycolytic flux, Krebs cycle activity, ATP levels, total biomass, $\mathrm{NAD}(\mathrm{P}) \mathrm{H}, \mathrm{Ca} 2+$, mitochondrial pH, membrane potential, redox state (Papkovsky and Zhdanov AV. 2021), the measure of changes in glycogen, lipid, and protein budgets (Smolders et al., 2003). It is noteworthy that the energy budget concerns not just cells, but also complex organisms: e.g., the number of calories in case of sporting activities can be quantitatively assessed.

Despite its qualities, RTS has some weaknesses with regard to its simplifying assumptions and rigid requirements. Less stringent accounts have been provided that consider less constrained situations, introducing, e.g., novel factors such as a moderate change with the external, opening the economy to trade, and so on. For a survey of the novel developments, see Koopmans (1965); Solow (1974); Mirrlees (1967), Arrow et al. (2012). For example, the Ramsey's model points to very high saving rates. According to Ramsey, over half of resources must be saved: i.e., an income of $£ 500$ per annum requires an amount that should be saved of about $£ 300$ per annum. Another thorny issue is that the passage of time for an individual is not the same as for generations, so that an individual's lifetime well-being is different from intergenerational well-being. RTS adopts the short-cut to provide the short-term and the long-term well-beings with the 
same functional form. Nevertheless, there is little evidence to suggest that such simplification holds in the real-world intergenerational life. Also, Ramsey assumed that time is a continuous and not a discrete variable, perhaps because the mathematics is simpler. In the last decades, many scholars provided efforts to overtake the limitations of the otherwise powerful Ramsey's theory. To show a feasible development, Dasgupta and Heal (1974) delivered a RTS extension including natural capital in production which, referred to our biological model, would make possible to introduce phenotypical, epigenetic, genotypical and evolutionary improvements in the cellular capabilities of labour and of energetic production.

At this point it comes to wonder: what for? Why should we provide an effort to correlate seemingly disparate fields such as the economic theory of saving and the cellular energy budget? The answer is straightforward: such comparison is very promising. Suggesting an interest in the distant future that is worth to be considered, RTS allows to calculate the amount of energy that a living individual must spare in order to optimize its chances to survive longer and in good health.

RTS's plot says how much energy must be spared from the available stock of the cellular budget to maximize the energy available for consumption. Indeed, high energetic consumption impairs the future well-being of the cell, while larger amount of reservoir might preserve the organism for longer times. RTS could be useful to compare the long-term effects of different energetic options. To provide a practical example, do the benefits of energetic expensive activities such as bodybuilding overcome the long-term damages? Does sport at competitive levels in children contribute to extend their well-being and lifetime? Furthermore, the knowledge of the proper quantity of cellular energy to store and spare might pave the way to novel anti-stress, beauty treatments and anti-ageing approaches.

The system-nation described by RTS reminds cell cultures, in which the parameters are strictly standardised and the energetic sources/flows carefully regulated. Ramsey's assumption that the community goes on forever in a world with an indefinite future lends itself well with the "infinite life" assumption adopted for cell colonies such as, e.g., Caco-2, the immortalized cell line of human colorectal adenocarcinoma cells (Marziano et al., 2019). In this peculiar case, the Ramsey's plot provides us with a useful information: how much energy must be provided to achieve the best preservation and longest survival of a cell culture to ensure the welfare of its future generations? In accordance with this line of reasoning, RTS might also contribute to improve the survival of beneficial bacterial colonies and biofilms, such as, e.g., endogenous saprophytic intestinal bacteria or exogenous probiotic mixtures (Giannetti et al., 2018), or to obliterate detrimental biofilms, such as, e.g., cardiac valvular colonies of harmful Streptococcal strains (Hosokawa et al., 2018).

We want to end with a more speculative insight. While individuals often save for their children, Ramsey asks how much should society save. In touch with this account, RTS implementation in the study of living organisms suggests that increases in intracellular energetic levels might lead to increases in stable phenotypes and decision-making capabilities. If the cells with sub-threshold intracellular energy are forced to adopt a singular phenotype and a specific cell fate (Tozzi and Peters, 2017; Peters et al., 2017; Tozzi et al., 2017), this could mean that energetic differences among different cells may help to explain the experimentally detected variability in cellular decision-making across biological systems. 


\section{REFERENCES}

1) Arrow KJ, Dasgupta P, Goulder LH, Mumford KJ, Oleson K. 2012. Sustainability and the Measurement of Wealth. Environment and Development Economics, 17(3), 317-355.

2) Attwell, D., \& Laughlin, S.B. (2001). An energy budget for signaling in the grey matter of the brain. Journal of Cerebral Blood Flow Metabolism, 21(10), 1133-1145.

3) Castro LC, Araujo AS 2019. Marginal Utility \& its Diminishing Methods. International Journal of Tax Economics and Management: 36-47.

4) Dasgupta P. 1969. On the Concept of Optimum Population. Review of Economic Studies, 36(3), $295-318$.

5) Ferral N, Gomez N, Holloway K, Neeter H, Fairfield M, et al. 2020. The extremely low energy cost of biosynthesis in holometabolous insect larvae. J Insect Physiol. 120:103988. doi: 10.1016/j.jinsphys.2019.103988.

6) Giannetti E, Maglione M, Alessandrella A, Strisciuglio C, De Giovanni D, et al. 2017. A Mixture of 3 Bifidobacteria Decreases Abdominal Pain and Improves the Quality of Life in Children With Irritable Bowel Syndrome: A Multicenter, Randomized, Double-Blind, Placebo-Controlled, Crossover Trial. J Clin Gastroenterol. 51(1):e5-e10. doi: 10.1097/MCG.0000000000000528.

7) Hosokawa S, Okayama H, Hiasa G, Kawamura G, Shigematsu T, et al. 2018. Isolated Left Atrial Infective Mural Endocarditis. Intern Med. 57(7):957-960. doi: 10.2169/internalmedicine.9559-17.

8) Kahneman D, Tversky A. 1979. Prospect Theory: An Analysis of Decision Under Risk. Econometrica. 47 (2): 263-292. doi:10.2307/1914185.

9) Kerr R, Jabbari S, Johnston IG. 2019. Intracellular Energy Variability Modulates Cellular Decision-Making Capacity. Sci Rep. 9(1):20196. doi: 10.1038/s41598-019-56587-5.

10) Koopmans TC. 1965. On the Concept of Optimal Economic Growth. Pontificiae Academiae Scientiarum Scripta Varia, 28. Reprinted in T.C. Koopmans (1966), The Econometric Approach to Development Planning (Amsterdam: North Holland).

11) Marziano M, Tonello S, Cantù E, Abate G, Vezzoli M, et al. 2019. Monitoring Caco-2 to enterocyte-like cells differentiation by means of electric impedance analysis on printed sensors. Biochim Biophys Acta Gen Subj. 1863(5):893-902. doi: 10.1016/j.bbagen.2019.02.008.

12) Misak C. 2020. Frank Ramsey: A Sheer Excess of Powers Copertina rigida - 13 febbraio 2020. OUP Oxford. ISBN-13: 978-0198755357.

13) Mirrlees JA 1967. Optimum Growth When Technology is Changing. Review of Economic Studies, 34(1), 95124.

14) Murphy CA, Nisbet RM, Antczak P, Garcia-Reyero N, Gergs A, et al. 2018. Incorporating Suborganismal Processes into Dynamic Energy Budget Models for Ecological Risk Assessment. Integr Environ Assess Manag. 14(5):615-624. doi: 10.1002/ieam.4063

15) Papkovsky DB, Zhdanov AV. 2021. Cell Energy Budget Platform for Multiparametric Assessment of Cell and Tissue Metabolism. Methods Mol Biol.2276:305-324. doi: 10.1007/978-1-0716-1266-8_23.

16) Parfit D. 1984. Reasons and Persons. Oxford: Oxford University Press.

17) Peters JF, Tozzi A, Ramanna S, Inan E. 2017. The human brain from above: an increase in complexity from environmental stimuli to abstractions. Cognitive Neurodynamics,11(4), 391-394. DOI: 10.1007/s11571-0-179428-2.

18) Ramsey FP. 1928. A Mathematical Theory of Saving. The Economic Journal. 38(152): 543-559.

19) Schmidt CA, Fisher-Wellman KH, Neufer PD. 2021. From OCR and ECAR to energy: Perspectives on the design and interpretation of bioenergetics studies. J Biol Chem. 297(4):101140. doi: 10.1016/j.jbc.2021.101140.

20) Sengupta, B., et al. (2013) Balanced excitatory and inhibitory synaptic currents promote efficient coding and metabolic efficiency. PLoS computational biology 9, e1003263.

21) Smolders R, De Boeck G, Blust R. 2003. Changes in cellular energy budget as a measure of whole effluent toxicity in zebrafish (Danio rerio). Environ Toxicol Chem. 22(4):890-9.

22) Solow RM. 1974. Intergenerational Equity and Exhaustible Resources. Review of Economic Studies, 41 (Symposium Issue), 29-45.

23) Song Y, Park JO, Tanner L, Nagano Y, Rabinowitz JD; Shvartsman SY. 2019. Energy budget of Drosophila embryogenesis. Curr Biol. 29(12):R566-R567. doi: 10.1016/j.cub.2019.05.025.

24) Tozzi A, Peters JF. 2017. From abstract topology to real thermodynamic brain activity. Cognitive Neurodynamics, 11(3) 283-292. Doi:10.1007/s11571-017-9431-7.

25) Tozzi A, Peters JF, Fingelkurts AA, Fingelkurts AA, Marijuán PC. 2017. Topodynamics of metastable brains. Physics of Life Reviews, 21, 1-20. http://dx.doi.org/10.1016/j.plrev.2017.03.001.

26) Zhdanov AV, Favre C, O'Flaherty L, Adam J, O'Connor R., et al. 2011. Comparative bioenergetic assessment of transformed cells using a cell energy budget platform. Integr Biol (Camb. 3(11):1135-42. doi: 10.1039/c1ib00050k. 